\title{
Pour en lire plus : Éduquer à l'urgence climatique.
}

\section{Claire Polo}

\section{(2) OpenEdition}

Journals

Édition électronique

URL : https://journals.openedition.org/ere/8315

ISSN : 2561-2271

\section{Éditeur}

Centr'ERE

Référence électronique

Claire Polo, «Pour en lire plus : Éduquer à l'urgence climatique. », Éducation relative à l'environnement [En ligne], Volume 17-1 | 2022, mis en ligne le 27 janvier 2022, consulté le 02 février 2022. URL : http:// journals.openedition.org/ere/8315

Ce document a été généré automatiquement le 2 février 2022

La revue Éducation relative à l'environnement est mise à disposition selon les termes de la Licence Creative Commons Attribution - Pas d'Utilisation Commerciale 4.0 International. 


\title{
Pour en lire plus : Éduquer à l'urgence climatique.
}

\author{
Claire Polo
}

\section{RÉFÉRENCE}

Anne-Françoise Gibert (2020). Éduquer à l'urgence climatique. Dossier de veille de l'Institut français de l'Éducation, $n^{\circ} 133,44$ pages

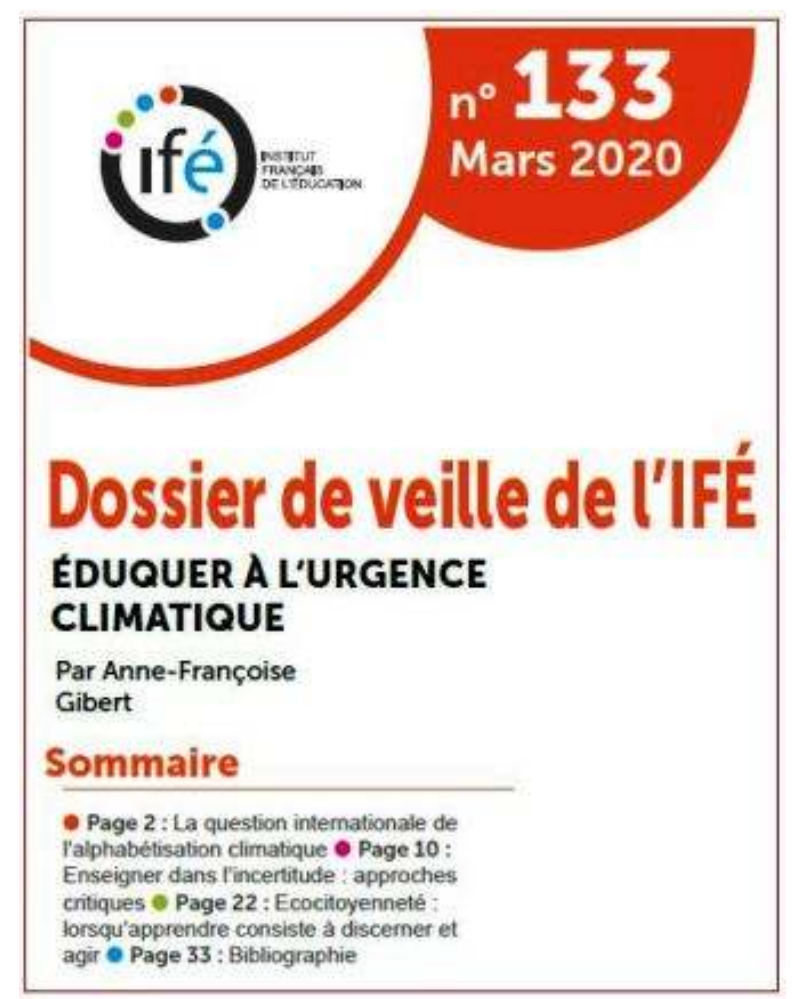


1 Cette revue de littérature sur l'éducation au changement climatique (ECC) propose un tour d'horizon assez large de ce champ en émergence, ici perçu depuis, et parfois à travers, la recherche francophone et centré sur l'école (éducation formelle). Elle constitue une synthèse précieuse pour qui désire découvrir à la fois l'état des recherches et des pratiques en la matière. Le sommaire ne traduit que partiellement la richesse du propos, structuré en trois parties: l'alphabétisation climatique, l'enseignement et l'écocitoyenneté.

2 La première partie discute des différents termes et concepts qui circulent autour de l'ECC et présente aussi les limites de l'approche d'alphabétisation climatique, qui serait plutôt une composante de l'alphabétisation scientifique, avec un accent sur les savoirs pratiques tournés vers l'action. Les parentés de l'ECC avec l'éducation à l'environnement, au développement durable, à la durabilité et à l'écocitoyenneté sont discutées, aux plans historique et conceptuel. L'autrice choisit de le faire via un focus sur l'alphabétisation énergétique, qui serait elle-même à la fois plus large que l'alphabétisation climatique (incluant des enjeux fondamentaux sans lien direct avec le réchauffement climatique) et plus restreinte (concernant une partie seulement des enjeux associés à l'urgence climatique).

3 Il est toutefois dommage que l'héritage disciplinaire très « scientifique » de l'ECC, du moins tel qu'il se dégage de ce dossier de veille, ne permette pas une véritable appréhension interdisciplinaire de l'alphabétisation énergétique. En effet, dans son recensement des idées erronées qui y font obstacle, l'autrice ne mentionne rien relevant des sciences humaines et sociales alors même que les aspects économiques, géopolitiques et juridiques sont fondamentaux pour comprendre les questions énergétiques (mais peut-être est-ce effectivement un angle mort de la recherche ?).

4 Par ailleurs, cette première partie du document aborde la délicate question du lien entre connaissances et comportements, en y incluant la dimension affective, trop souvent déconsidérée dans les cultures (et les écoles !) occidentales, mais pourtant déterminante pour la formation des attitudes concrètes. L'auteure pointe également la question de l'importance du milieu social et familial, au-delà du rôle de l'école, ainsi que des politiques publiques. À mon sens, on touche ici à la difficulté principale de l'action éducative dans ce domaine, à savoir une posture générationnelle globale reposant sur un message du type «fais ce que je dis, pas ce que je fais ", alors même que toute éducation et toute socialisation fonctionnent en grande partie sur l'imitation. Moralement, comment exiger de nos enfants qu'ils intègrent à la fois les codes de nos sociétés développées pour s'y engager activement, et qu'ils gardent une distance critique vis-à-vis du bonheur matériel qu'elles promeuvent? Comment créer le renoncement à des pratiques qui sont monnaie courante pour la grande majorité des adultes d'aujourd'hui, en particulier parmi les sphères de pouvoir (manger de la viande, prendre l'avion, avoir le téléphone dernier cri, etc.) ? Comment inciter la jeune génération à adopter un "espoir constructif " plutôt qu'une posture de déni ou une anxiété paralysante quand on lui offre principalement, à grande échelle, un modèle d'inaction?

5 Les deux parties suivantes de cette revue de littérature offrent des pistes de réponses. Sous le terme général d' " approches critiques ", des pratiques et dispositifs variés sont présentés comme autant de modalités d'enseignement de ce que l'on pourrait qualifier d'autodéfense intellectuelle vis-à-vis de la pensée dominante qui mène aux catastrophes annoncées. Sont ainsi listés des éléments relevant de l'éducation aux 
médias, aux controverses (sur les actions à entreprendre, le réchauffement à l'œuvre relevant du consensus scientifique), et à l'esprit critique, notamment via le dialogue philosophique. Au-delà de l'apprivoisement des incertitudes sur les effets du changement climatique et des mesures prises pour l'atténuer et s'y adapter, il s'agit de rétablir les valeurs comme objets d'enseignement légitimes. Il me semble que réside ici un enjeu démocratique de taille: une action éducative ne saurait rimer avec émancipation plutôt qu'endoctrinement que si les valeurs souvent implicites des discours dominants peuvent être mises au jour et débattues. En effet, prétendre que le consensus actuel est souhaitable revient à court-circuiter toute possibilité d'imaginer un avenir réellement autre, alors même que l'idéal de justice sociale, par exemple, n'est pas le premier moteur du fonctionnement de nos systèmes socio-économiques. Cependant, il est désolant de constater avec l'autrice l'écart encore important entre ces préconisations et la majorité des pratiques d'ECC, fondées sur la responsabilisation individuelle via l'inculcation d'écogestes, mais faisant l'impasse sur les enjeux politiques plus larges.

6 Pour les enseignant.e.s comme pour les élèves, une véritable formation à l'ECC devrait prendre appui sur des dilemmes incarnés dans des contextes locaux, où des actions tangibles sont possibles. C'est principalement cette perspective " communautarienne » qui est mise en avant dans la dernière partie du dossier, comme occasion d'expérimenter à l'école un engagement collectif participant à l'amélioration concrète de l'« habitabilité » de son propre territoire. Seul un tel empowerment permettrait de vivre des émotions constructives, nourrissant des récits véritablement nouveaux et centrés sur un futur désirable, alors que l'heure est à l'« écoanxieté » et à la " solastagie ", et que la crise sanitaire a déjà des répercussions sur la santé psychique des enfants et des jeunes. Un apprentissage expérientiel, notamment hors des murs de la classe, au contact direct du monde social et des écosystèmes, mais aussi, paradoxalement, en plongeant dans le pouvoir de la fiction artistique (dont on nous disait il y a peu ne pas être essentielle en tant de crise), serait à même de développer une pensée prospective et ouvrir l'horizon devant nous.

7 Si un tel tableau d'ECC transformative est réjouissant, la plausibilité de sa mise en place à l'école est toutefois questionnée. À juste titre, l'autrice pointe le facteur clef de l'environnement institutionnel pour un tel programme. Ainsi, c'est seulement en rendant dès aujourd'hui les institutions critiques et résilientes que l'on peut former les écocitoyen.ne.s de demain. L'ECC devenant à la fois le moyen et la fin: avoir la cohérence, déjà, de transformer l'école, pour qu'elle puisse aider à transformer le monde.

8 Reste que toute la sociologie de l'école publique montre son fonctionnement conservateur et sa propension à reproduire la société à l'identique plutôt qu'à la faire évoluer. D'ailleurs, il est dommage que cette discipline soit relativement peu représentée dans ce dossier, par rapport à la psychologie, par exemple. On apprend toutefois que les femmes, les jeunes, les peuples autochtones, les personnes habitant des régions directement menacées, voire déjà sinistrées par le réchauffement climatique y sont plus sensibles, et plus enclin.e.s à agir pour prévenir et réduire les impacts du changement climatique. Dommage que les décisions soient encore principalement prises, à l'échelle du globe, par des hommes plus âgés, de zones plutôt épargnées à court terme, et dénuées de culture holistique. Y a-t-il encore un espoir de les éduquer? 
9 Ce dossier thématique est en libre accès: http://veille-et-analyses.ens-lyon.fr/DAVeille/133-mars-2020.pdf

\section{AUTEUR}

\section{CLAIRE POLO}

Université Lyon 2, France. 